\title{
EDITORIAL SPECIAL ISSUE ON ARTIFICAL INTELLIGENCE AND INFORMATION SYSTEMS ON KNOWLEDGE, TECHNOLOGY AND SERVICE MANAGEMENT
}

\author{
Xijin Tang ${ }^{1,2}$ Jiangning $\mathrm{Wu}^{3}$ \\ ${ }^{I}$ Academy of Mathematics and Systems Science, Chinese Academy of Sciences, Beijing 100190, China \\ xjtang@iss.ac.cn $(\bowtie)$ \\ ${ }^{2}$ University of Chinese Academy of Sciences, Beijing 100049, China \\ ${ }^{3}$ Faculty of Management and Economics, Dalian University of Technology, Dalian, 116024, China \\ jnwu@dlut.edu.cn (囚)
}

The annual International Symposium on Knowledge and Systems Sciences aims to promote the exchange and interaction of knowledge across disciplines and borders to explore the new territories and new frontiers. The past 18-year continuous endeavors since 2000 are illustrations that knowledge science and systems science can complement and benefit each other methodologically when studying and solving a variety of problems. During November 17-19, 2017, the 18th International Symposium on Knowledge and Systems Sciences (KSS2017) was held in Bangkok under the theme of "Artificial Intelligence and Information Systems for Knowledge, Technology and Service Management".

This special issue contains 8 extended papers originally presented at KSS2017. The topics cover knowledge discovery, knowledge management, knowledge systems engineering, big data analytics, collective intelligence, social systems sciences and advanced machine learning.

Even on an ever-lasting topic in recent KSS, Guo, Du and Kou (2018) proposed a new ranking method through online reviews based on different aspects of the alternative products, which combines both objective and subjective sentiment values. Firstly, weights of these aspects were determined with LDA topic model to calculate the objective sentiment value of the product. A directed graph was constructed to aggregate heterogeneous online reviews, and the final score of each product was computed by an improved PageRank algorithm considering not only the information mining from the massive online reviews but also consumer's preference. A case study was given to illustrate the feasibility and effectiveness of the proposed method which targets to make personalized recommendation. The Spearman coefficient is used to demonstrate that our experimental results have a strong correlation with actual sales ranking.

Extended from the most downloaded paper among all KSS2017 papers, Ni and Liu (2018) proposed a conceptual framework to design and implement a model of iterative inquiry for an automated information broker that bridges clients with a service provider. As a chatbot interacting with the client, the developed automated agent accomplishes a sequence of tasks including natural language inquiry, 
knowledge gathering, reasoning, and responding. The framework combined a question-answering reasoning mechanism while utilizing domain-specific knowledge base. A prototype, Mandy, is designed and implemented for healthcare services.

Originally as one of the invited keynote speeches, Gloor et al. (2018) proposed a methodology to build an automated mood tracking system. They measured and predicted states of Activation and Happiness using a body sensing application connected to smart watches. Through the sensors of commercially available smart watches they collect individual mood states and correlate them with body sensing data such as acceleration, heart rate, light level data, and location through the GPS sensor built into the smart phone connected to the smart watch. Mood state is measured by asking users on the smart watch four times per day for seven weeks. The research found that both Happiness and Activation are negatively correlated with heart beats and with the levels of light. People tend to be happier when they are moving more intensely and are feeling less activated during weekends. The research also found that people with a lower Conscientiousness and Neuroticism and higher Agreeableness tend to be happy more frequently.

Instead of concentrating on personal state, $\mathrm{Xu}$ and Tang (2018) concern more on societal state. Based on the Baidu hot news search word (HNSW) derived societal risk perception, they first studied the relationships between search trends and societal risk perception, then studied the relationships between societal risk perception and stock volatility by three different econometric methods. They found that there existed causal relations between Baidu Index and societal risk perception; the perception of finance \& economy, social stability, and government management has distinguishing effects on the volatility of both Shanghai Composite Index and Shenzhen Composite Index. In addition, the weekend and holiday effects of societal risk perception on the stock market are verified. The research demonstrated that capturing societal risk based on on-line public concerns is feasible and meaningful.

Dong et al. (2018) proposed an ex-ante forecasting theory related to the Internet public opinion caused by the failure of public policies. The corresponding model is used to find factors that may influence the duration of Internet public opinions related to the public policy itself and the issues about the context when and where the policy is released. 23 typical Internet public opinion cases caused by the failure of public policies were studied and 9 prediction models were proposed by applying the multivariate linear regression model, multivariate nonlinear regression model, and the Cobb-Douglas function.

Wang, Dang and $\mathrm{Wu}$ (2018) proposed a novel computational experiment method to determine the task allocation schemes to achieve optimal performance for a knowledge-intensive team. They built a knowledge-intensive team system model based on complex adaptive system theory and agent modeling technology, designed task allocation strategies and a team performance measurement scale utilizing computational experiment, and analyzed how different task allocation strategies impact the different performance indicators. The experimental results showed that the recommend task allocation strategies vary under different 
conditions, such as the knowledge levels of members, team structures, and tasks to be assigned, particularly when the requirements to the team are different.

Pan, Xia and Luo (2018) attempted to disclose the implications for social marketing strategies through a series of computational simulations toward the dynamics of brand acceptance by a coupled agent-based dynamic model which combines the Majority-Rule-based Voter model in opinion dynamics with the SI Model for information spreading. Two parameters, the decayed transmission rate and the diffusion frequency, are focused. In the absence of opinion interaction, the simulation results indicated that when a brand tries to occupy a larger market share through social marketing approaches, it is always effective to let the opponent to be the propaganda target. While with the Majority-Rule-based Voter Model included, they observed that the opinion interaction could have a dual function, illustrating that a brand holding a small market share in the first place needs to adopt diverse marketing approaches according to different marketing environment types.

Pannakkong, Sriboonchitta, and Huynh (2018) proposed an ensemble model composed of autoregressive integrated moving average (ARIMA), artificial neural network (ANN), restricted Boltzmann machines (RBM), and discrete wavelet transform (DWT) to improve the prediction accuracy of time series forecasting. The forecasting capability of the ensemble model was tested with three well-known time series: sunspot, Canadian lynx, exchange rate time series. The prediction performance was compared to the other six forecasting models. The results indicated that the proposed ensemble model produced the best performance in all three data sets and all three measures namely MSE, MAE and MAPE.

As addressed, the selected 8 papers employ the derived knowledge to tackle various issues coming from different management practices such as service/business management, societal management, and team management, etc. Except the invited paper (reviewed for journal publication only), each selected paper was not only reviewed by at least one original KSS2017 reviewer, but a new reviewer was invited, and thus great modifications were taken. We hope this special issue presents the main endeavors of KSS community toward the complicated issues.

\section{References}

[1] Dong, X.F., Lian, Y., Li, D. \& Liu Y.J. (2018). The application of cobb-douglas function in forecasing the duration of internet public opinions caused by the failure of public policies. Journal of Systems Science and Systems Engineering, 27(5):632-655.

[2] Gloor, P.A., Colladon, A.F., Grippa, F., Budner, P. \& Eirich, J. (2018). Aristotle said "Happiness is a state of activity" - Predicting mood through body sensing with smartwatches. Journal of Systems Science and Systems Engineering, 27(5): 586-612.

[3] Guo, C.H., Du, Z.L. \& Kou, X.Y. (2018). Products ranking through aspect-based sentiment analysis of online heterogeneous reviews. Journal of Systems Science and Systems Engineering, 27(5): 542-558.

[4] Ni, L. \& Liu, J.M. (2018). A Framework for domain-specic natural language information 
brokerage. Journal of Systems Science and Systems Engineering, 27(5): 559-585.

[5] Pan, Q., Xia, H.X. \& Luo, S.L. (2018). A coupled dynamic model of brand acceptance and promotive information spreading. Journal of Systems Science and Systems Engineering, 27(5): 677-689.

[6] Pannakkong, W., Sriboonchitta, S. \& Huynh, V.N. An ensemble model of ARIMA and ANN with restricted Boltzmann machine based on decomposition of discrete wavelet transform for time series forecasting. (2018). Journal of Systems Science and Systems Engineering, 27(5): 690-708.

[7] Wang, S.N., Dang, Y.Z. \& Wu, J.N. (2018). How task allocation strategy affects team performance: a computational experiment. Journal of Systems Science and Systems Engineering, 27(5): 656-676.

[8] Xu, N. \& Tang, X.J. (2018). A causality analysis of societal risk perception and stock market volatility in China. Journal of Systems Science and Systems Engineering, 27(5): 613-631.

Xijin Tang is a full professor in the Academy of Mathematics and Systems Science, Chinese Academy of Sciences. She received her BEng (1989) on computer science and engineering from Zhejiang University, MEng (1992) on management science and engineering from University of Science and Technology of China and $\mathrm{PhD}$ (1995) from CAS Institute of Systems Science. Her recent interests are meta-synthesis and advanced modeling, opinion mining and opinion dynamics, social computing, decision support systems, etc. She is one of vice presidents and secretary in general of International Society for Knowledge and Systems Sciences (ISKSS) and takes charge of program committee of annual KSS symposium. Currently she is vice editor-in-chief of Chinese Journal of Systems Engineering and also members of editorial boards of Journal of Systems Science and Complexity (Springer), Systema: connecting matter, life, culture and technology, and Journal of Systems Science and Mathematics (Chinese).

Jiangning $\mathbf{W u}$ is a full Professor in the Faculty of Management and Economics at Dalian University of Technology. She received her B. Eng. and MPhil degrees in 1986 and 1989 respectively from Dalian University of Technology, China. She obtained her Ph.D. degree in 2001 from the University of Hong Kong in Artificial Intelligence. Now she is serving as Associate Editor for International Journal of Knowledge and Systems Sciences (IJKSS). She is a reviewer of a number of domestic and foreign periodicals.

She has research interests in system engineering, knowledge management, data mining, and complex systems. In the past years, she has completed some projects from NSFC, local government, and enterprises covering the strategic analysis of regional economic development, knowledge creation in working teams, the big data analytics, recommender systems in electronic commerce. She published more than 40 papers in journals as well as 7 books/chapters both in Chinese and English, and more than 60 contributions in International Conferences related to her areas. 